\title{
SPL Business Operation Qualifier Terminology
}

National Cancer Institute

\section{Source}

National Cancer Institute. SPL Business Operation Qualifier Terminology. NCI Thesaurus.

Code C101885.

Terminology used to qualify the information on business operations concerned with medical product development, manufacturing, and marketing in the framework of the Structured Product Labeling documents. 